88071746

\title{
BIOLOGY AND CONSERVATION OF THE CORAL PINK SAND DUNES TIGER BEETLE, CICINDELA ALBISSIMA YEAR 2008, FINAL REPORT
}

\section{Part I. POPULATION AND BIOLOGICAL STUDIES IN 2008 AND PREVIOUS YEARS \\ Part II. POPULATION VIABILITY ANALYSIS OF C. albissima and IMPLICATIONS FOR MANAGEMENT}

FINAL REPORT

To: BLM, Utah State Office 440 West 200 South, Suite 500

Salt Lake City, UT 84145-0155

From: C. Barry Knisley and Charles Gowan

Department of Biology

Randolph-Macon College

Ashland, VA 23005

Date: APRIL 22, 2009 


\begin{abstract}
PART I. This report presents results of continuing studies of the Coral Pink Sand Dunes Tiger Beetle funded by a Challenge Cost Share Agreement between the Bureau of Land Management and Randolph-Macon College. Included in this report is new information obtained in the year 2008 and an updated population viability analysis. The total adult population size estimate of C. albissima for 2008 was 1072, with a 95\% confidence range of 959 to 1185 . This count represents a significant increase from the 700 in 2007 but a decline from the previous even year cohort estimate of 1112 in 2006 . The 2008 estimate also indicates a 6-year period of counts of 1112 or less since the highest ever count of 2944 was recorded in 2002. This 6-year period also includes all but one of the lowest counts since counts were initiated in 1992. The densities and distribution pattern among the individual swales were generally similar to that in most previous years. Highest adult numbers were in swales $J K$ with 280 adults, 2-3 with 171, $H$-WH with 161, 1 with 118 , public with 63 and $Q R / E Q R$ with 107. As has been the norm in low abundance years, numbers of adults in swales outside of the primary core area were very low: only 15 adults were found in all swales north of swale 4 and only 11 adults were in the D swales. Only two adults were counted in the AAA-AA area at the far northern end of the dune field, but larval numbers in these northern swales were higher (182 total) than in other recent years. The total percent of the population within the protected swales (within the conservation area) was $80 \%$, continuing the pattern seen in all years since implementation of the conservation agreement. Continued monitoring of rainfall totals at the Kanab station has continued because of the importance of rainfall in driving population trends. The rainfall amounts, both total for April through October (the period of adult and larval activity) and April-June (the critical period for oviposition and recruitment) were the lowest ever in 2008, 2.82 inches and 0.22 , respectively. This compares with the previous low April-October total of 5.15 in 1993 and the previous low April-June total of 0.41 in 2002. It is also notable that the totals for 2006, 2007 and 2008 were significantly lower than any other three-year period, and consequently project continuing low adult numbers for at least the next two years.
\end{abstract}

PART 2. The new PVA is an updated version of the 1999 one and considered to be more reliable because it includes more years of data (10 versus 7) and because the population estimates in these past 10 years are much more accurate. The primary result of this PVA is that the CPSD Tiger Beetle continues to be at risk of extinction. If the past 10 years parameters are used (growth rate of 1.058, standard deviation of growth rate $=$ stochasticity of .646 , carrying capacity of 3500 adults, starting population of 1200 ), the probability of the population becoming extinct is .32 and the probability of the population declining to 50 individuals is .87. However, substituting a growth rate of 1.35 while keeping the other values the same reduces the extinction probability to .001 and to 50 individuals to .003 . This comparison demonstrates the major effect of growth rate on extinction risk. The growth rate over the 10 years was 1.058 , based on positive growth rates in only three years $(1.25,1.47,2.32)$; all other years had negative rates, thus producing a low mean growth rate. The only other parameter which had a significant effect on the resulting extinction risk is stochasticity. If this value is set at .33 , extinction probability is very low in most scenarios, but if it is increased to .95 (a value that was seen among some of the years) extinction risk increases to near 1.0. These results suggest 
that long term survival of the CPSD tiger beetle population will most likely occur if growth rate of the population is higher than the mean for the past 10 years (which includes 7 years of negative growth). Management options to increase growth rate are C. 2 limited since we believe that climate, especially rainfall and soil moisture, is the main factor affecting growth rate. Although carrying capacity increase had only a limited effect, increasing the amount of protected area, supplementing moisture in localized patches, or reducing vegetation in selected swales might increase available habitat, and should be considered on an experimental basis.

\section{PART I. POPULATION AND BIOLOGICAL STUDIES}

\section{INTRODUCTION}

The Coral Pink Sand Dunes Tiger Beetle, Cicindela albissima, is known only from the Coral Pink Sand Dunes in southern Utah. Studies on this rare insect were begun in 1991 when a population census and other preliminary studies were conducted and suggested its probable rarity. Several years later, a taxonomic study of this beetle and its relatives in the Cicindela martima group using mitochondria DNA indicated this beetle was sufficiently distinct from the other subspecies of Cicindela limbata that it should be considered a separate species. Studies on its biology, especially the population dynamics, have been carried out each year since those initial studies. These studies have documented a highly variable population size for C. l. albissima since 1992, with numbers ranging from a peak of 2944 in 1996 to a low count of 558 in 2005. The report presents results of our continuing studies with this rare beetle at Coral Pink Sand Dunes, with support from a Utah BLM Challenge Cost Share Program. Specific research objectives for the 2008 field season included continued monitoring population size throughout the dune field, studying impact of rainfall and soil moisture on population dynamics, and developing a Population Viability Analysis using new information obtained since 1998 .

\section{METHODS}

\section{Adult Surveys}

Throughout the years of this study, monitoring adult population size has been a primary major research goal, and again in 2008 this was continued in the same occupied habitat of the dunes using the same method as in previous years. Knowing the population size in each year is important for documenting the trend in numbers over time and as an indicator of the long-term viability of the beetle population and health of the habitat at CPSD. The adult surveys in 2008 were done from May 13-24, the same time period as in previous years. The method we used to determine adult size in 2008 and since 1999 was the removal method. To obtain accurate results, the survey work needs to be carried out during periods of peak adult activity: sunny and warm, when sand surface temps are warm enough to initiate adult activity and not too hot to be unfavorable. On most days, 
these optimum conditions for conducting the removals occurred between 930 and 1230$1330 \mathrm{~h}$ and again from about $1630-1830 \mathrm{~h}$. On sunny days when surface temperatures increase to high levels ( $>45-50 \mathrm{C}$.), adults begin digging burrows to escape the unfavorable conditions. They typically re-emerge about $1630-1830$ for another 2 hour activity period before digging burrows again to spend the night

The usual procedure for conducting the removal method involves three workers systematically moving slowly through the area of one swale and collecting all of the adults that are observed. Typically, a small number of the adults in some swales are missed, flew off, or otherwise not captured. We have estimated that this loss accounts for only about $5 \%$ of the population. After completing the first search and capture in a swale, the captured adults were counted, placed in individual vials and retained in a cooler with ice. We then repeated the process a second, third and sometimes fourth time until we captured decreasing numbers with each pass. Collected adults were placed in vials and stored in the cooler after each pass. After the last pass all of the adults were released throughout the swale areas where they were collected. We then moved on to another swale and repeated the process if conditions were suitable. For many of the swales that had few adults $(<10-15)$ we made only one pass and used this number as the estimate for that swale. The removal results for each swale were analyzed using Program CAPTURE to produce population estimates and associated variances with totals from index counts added in to produce a total estimate for the population and the $95 \%$ confidence intervals. After each swale was sampled we walked the perimeter of the specific area sampled with a GPS and subsequently produced a GIS map of the whole survey area. From these maps and the survey data we computed densities of adults per swale so we could evaluate the habitat quality of swales for supporting beetles. Additional surveys using the visual index method were also carried out in several swales in area AAA and AA at the north end of the dune field and in area D to the southwhere adults have been found in previous surveys.

\section{Rainfall}

In this report we include monthly rainfall records from Kanab for the months that tiger beetle adults and larvae are active (April through October) for the years 1992 through 2008. We use the Kanab station data because it provides the most complete record and because the rainfall gauge at Coral Pink Sand Dunes State Park has been periodically non-functional. Rainfall and the coincidental soil moisture are believed to be the most important factor driving the population dynamics of the CPSD tiger beetle because increased rainfall/moisture has significant positive effects on many life history parameters, including oviposition, recruitment, survivor, development rate, and prey abundance.

\section{Larval Surveys and Translocation}

We did not conduct larval (or adult) translocations in either May or September of 2008 because of the small population size of adults and the corresponding small numbers of larvae. 
A major part of the 2008 study was conducting a population viability analysis (PVA) for the CPSDTB. This analysis was previously done in 1999 based on 1992-1998 population estimates but was redone because much additional population information has been obtained and our population estimates since that time have been more accurate. The analysis used the same basic approach as the previous PVA but included new input parameters. This study is included in Part 2 of this report.

\section{RESULTS AND DISCUSSION}

The total adult population size estimate of $C$. albissima for 2008 was 1072 , with a $\mathbf{9 5 \%}$ confidence range of 959 to 1185 . This count represents a significant increase from the 700 in 2007, but a decline from the previous even year cohort estimate of 1112 in 2006 (Fig. 1). The 2008 estimate also indicates a 6 year period of counts of 1112 or less since the highest ever count of 2944 in 2002. Moreover, these counts include a much higher proportion of lowest counts for any time since counts were initiated in 1992. Although many of the earlier counts (1992-1998) were determined to be overestimates, the accurate estimates from 1999-2001 $(1804,1270,1459)$ all were higher than any estimates in the past six years. We have attributed these population dynamics and the recent low counts to the significantly reduced rainfall during this period (see below).

Fig. 1. Population estimates of CPSD Tiger Beetle based on index counts (all years) and other methods (mark-recapture method, 1992-1998 and removal method, 19992008). Studies have indicated values for 1992-1998 overestimated population size while those from 1999-2008 are reliable estimates.

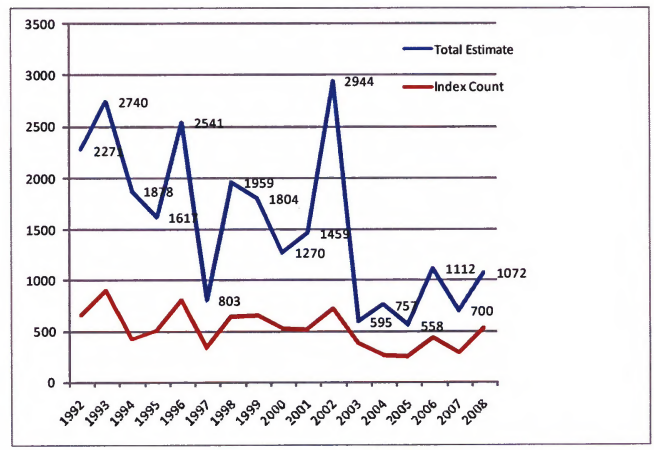


The densities and distribution pattern of adults among the individual swales were generally similar to most of the previous results (Fig. 2, Table 1). Highest adult numbers were in swales JK with $280,2-3$ with 171 , H-WH with 161 , 1 with 118 , public with 63 and $\mathrm{QR} / \mathrm{EQR}$ with 107. As has been the norm in low abundance years, numbers of adults in swales outside of the primary core area were very low. Only 15 adults were found in all swales north of swale 4 and only 11 adults were in the D swales which are south of the conservation area. Numbers of adults and larvae also generally low in the far northern part of the dune field. Adult numbers have always been very low, typically 5-15 total in this area and only 2 were counted in 2008 (both in AAA-2). Larval numbers in these northern swales have been more variable and higher than adult numbers, ranging from 452 in 1999 to 0 in 2005 and 2006 (Fig. 3). The larval count in 2008 was 182, 160 of these in AAA-2: 2 first instars, 52 seconds, and 128 thirds. This relatively high and increasing number of larvae in the past two years is unusual since previously higher numbers in the northern swales have been associated with higher rainfall and higher adult numbers in the main population. Possibly there may have been more dispersal and/or higher rainfall in this area in 2007, which would account for the higher larval numbers (especially third instars) in 2008.

Table 1. Results of 2008 Removal Method Estimates and Index Counts for CPSD Tiger Beetle in individual swales. $N$-hat is the number estimated for the respective swales.

\begin{tabular}{|c|c|c|c|c|c|c|c|c|}
\hline \multicolumn{9}{|c|}{$2008 \quad$ RESULTS } \\
\hline \multicolumn{9}{|c|}{ THREE PASS SWALES } \\
\hline & \multicolumn{3}{|c|}{ Pass } & \multirow[b]{2}{*}{ Total } & \multirow[b]{2}{*}{ N-hat } & \multirow[b]{2}{*}{ SE } & \multirow[b]{2}{*}{ Var } & \\
\hline Swale & 1 & 2 & 3 & & & & & \\
\hline Public & 24 & 15 & 10 & 49 & 63 & 11.207 & 125.601 & \\
\hline JK & 178 & 65 & 24 & 267 & 280 & 5.4263 & 29.445 & \\
\hline E2\&3 & 13 & 3 & 1 & 17 & 17 & 0.5312 & 0.282 & \\
\hline E1\&4 & 12 & 9 & 9 & 30 & 60 & 42.7066 & 1823.854 & \\
\hline $\mathrm{HWH}$ & 94 & 35 & 20 & 149 & 161 & 6.1359 & 37.649 & \\
\hline 1 & 56 & 28 & 17 & 101 & 118 & 9.2702 & 85.937 & \\
\hline WY & 21 & 11 & 6 & 38 & 43 & 4.6315 & 21.451 & \\
\hline TOTAL & 398 & 166 & 87 & 651 & 742 & NA & 2124.219 & \\
\hline \multicolumn{9}{|c|}{ FOUR PASS SWALES } \\
\hline & \multicolumn{3}{|c|}{ Pass } & & & & & \\
\hline Swale & 1 & 2 & 3 & 4 & Total & N-hat & $\mathrm{SE}$ & Var \\
\hline $2 \& 3$ & 52 & 54 & 31 & 16 & 153 & 171 & 9.7979 & 95.999 \\
\hline QR & 22 & 16 & 16 & 11 & $\begin{array}{c}65 \\
0\end{array}$ & 107 & 33.1424 & $\begin{array}{c}1098.4 \\
0\end{array}$ \\
\hline TOTAL & 74 & 70 & 47 & 27 & 218 & 278 & NA & 1194.4 \\
\hline \multicolumn{3}{|c|}{$\begin{array}{l}\text { Total beetles handled } \\
\text { Total Estimate } \\
\text { Confidence limits: }\end{array}$} & $\begin{array}{r}869 \\
1072 \\
959 \text { tc }\end{array}$ & 1185 & & & & \\
\hline
\end{tabular}




\begin{tabular}{|c|c|c|c|}
\hline Swale & N-hat & SE & Var \\
\hline Public & 63 & 11.2072 & 125.60133 \\
\hline JK & 280 & 5.4263 & 29.444732 \\
\hline E2\&3 & 17 & 0.5312 & 0.2821734 \\
\hline E1\&4 & 60 & 42.7066 & 1823.8537 \\
\hline $\mathrm{HWH}$ & 161 & 6.1359 & 37.649269 \\
\hline 1 & 118 & 9.2702 & 85.936608 \\
\hline WY & 43 & 4.6315 & 21.450792 \\
\hline 283 & 171 & 9.7979 & 95.998844 \\
\hline QR & 107 & 33.1424 & 1098.4187 \\
\hline Total: & 1020 & & 3318.636 \\
\hline \multicolumn{4}{|c|}{ Index Count Swales } \\
\hline Swale & Estimate & & \\
\hline $\mathrm{LM}$ & 1 & NA & Index Count \\
\hline $\mathrm{N}$ & 1 & NA & Index Count \\
\hline OP & 3 & NA & Index Count \\
\hline 1 & 2 & NA & Index Count \\
\hline DT & 3 & NA & Index Count \\
\hline$E 6 \& 7$ & 2 & NA & Index Count \\
\hline 4 & 17 & NA & Index Count \\
\hline 5 & 7 & NA & Index Count \\
\hline 9 & 0 & NA & Index Count \\
\hline $6 \& 7$ & 5 & NA & Index Count \\
\hline 8 & 3 & NA & Index Count \\
\hline ST & 2 & NA & Index Count \\
\hline $\mathrm{U}$ & 0 & NA & Index Count \\
\hline $\mathrm{V}$ & 2 & NA & Index Count \\
\hline$x$ & 2 & NA & Index Count \\
\hline $\bar{Y}$ & 1 & NA & Index Count \\
\hline WX & 1 & NA & Index Count \\
\hline & 52 & & \\
\hline
\end{tabular}


Fig.2. Map showing location of individual swales at CPSD. Note that legend is a typical density pattern and not specific for 2008 results.

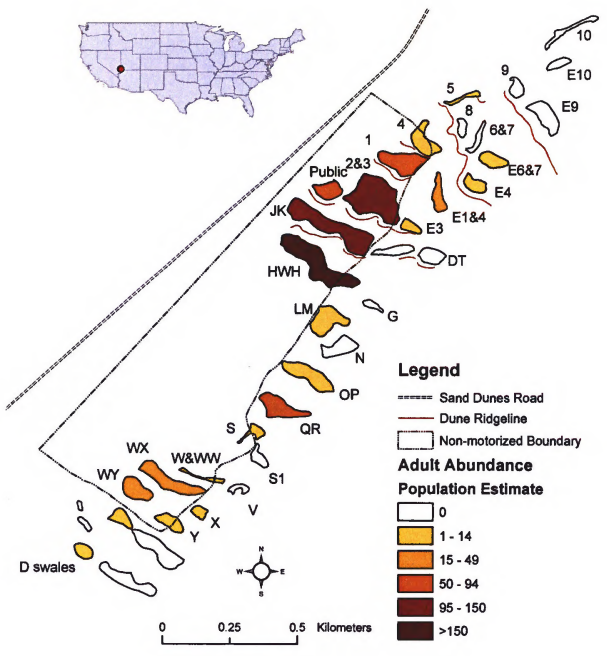


Fig. 3. Numbers of larvae counted in Conservation Area B (swale AA-2 and total for all swales) at the north end of the CPSD dune field, 1996-2008.

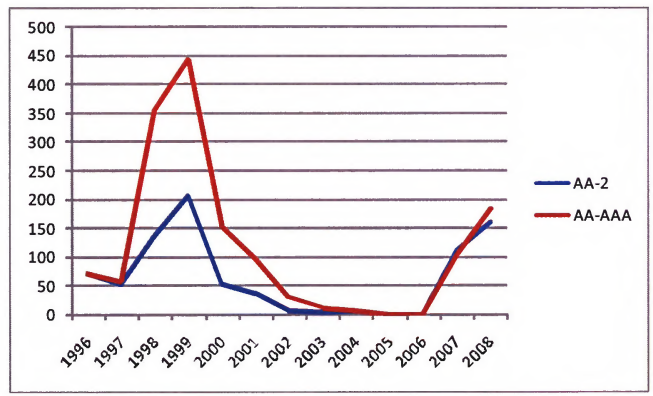

\section{Adults in Protected Versus Unprotected Swales}

Table 2 presents the numbers of adults in individual protected swales (within the conservation area) versus numbers in the unprotected swales outside the conservation area (see Fig. 2 for locations). The relative percents in these two areas in 2008 were very similar to the values of most recent years. In 2008, $80 \%$ were within Conservation Area $\mathrm{A}$ and $20 \%$ were in the unprotected swales outside the Conservation Area. Prior to the implementation of the Conservation Agreement (1992 to 1998), the percent in the currently protected swales were $70 \%$ or less. This pattern of a high percent within the protected swales suggests that the conservation area with its protected swales is very important in supporting the core of the population, especially during years when the total population is low. The Conservation Area not only protects adults and larvae from OHV activity, but includes the highest quality swales for recruitment and adult activity. Consequently, this protection reserve provides the best conditions for protection of most of the core population and can thus allow for higher growth rates and associated dispersal to other areas when more favorable climatic conditions occur.

Numbers of adults have been variable within individual swales as have adults over the past 10 years, but some swales have experienced changes independent of overall population trends. Swale 4 has supported many few adults since 2001, compared to years before that time. The only notable change has been increasing vegetation and 
coincidental stabilization of portions of the swale. This may have reduced oviposition/recruitment areas and subsequently adult numbers. Estimates in swales 2-3 have also declined but less significantly, perhaps as a result of a similar increase in vegetation cover. Among the unprotected swales, adult numbers have exhibited a pattern of decline in swales ST, E-4,6, E-6,7, swales N of swale 4 (5-9), OP and LMN. Vegetation increase cannot explain the general pattern of decline in these swales, but perhaps OHV impact or other factors are involved.

Table 2. Numbers of adults in swales protected within Conservation Area A versus those outside this area, since 1998 when protection area was established.

\begin{tabular}{|l|c|c|c|c|c|c|c|c|c|c|c|c|}
\hline Swale & \multicolumn{1}{|c|}{1998} & 1999 & $\mathbf{2 0 0 0}$ & $\mathbf{2 0 0 1}$ & $\mathbf{2 0 0 2}$ & $\mathbf{2 0 0 3}$ & $\mathbf{2 0 0 4}$ & $\mathbf{2 0 0 5}$ & $\mathbf{2 0 0 6}$ & $\mathbf{2 0 0 7}$ & $\mathbf{2 0 0 8}$ \\
\hline CONSERVATION AREA SWALES & & & & & & & & \\
\hline 4 & 46 & 98 & 23 & 62 & 74 & 20 & 5 & 9 & 28 & 4 & 17 \\
\hline 1 & 52 & 142 & 87 & 116 & 205 & 48 & 75 & 72 & 76 & 45 & 118 \\
\hline pub & & 76 & 97 & & 275 & 36 & 65 & 6 & 155 & 60 & 63 \\
\hline 2,3 & 155 & 213 & 244 & 278 & 349 & 108 & 111 & 144 & 148 & 140 & 171 \\
\hline IJK & 91 & 266 & 189 & 191 & 748 & 114 & 114 & 91 & 265 & 143 & 280 \\
\hline HWH & 59 & 369 & 133 & 180 & 386 & 90 & 179 & 111 & 178 & 114 & 161 \\
\hline W-WW & 31 & 18 & 3 & 7 & 15 & 2 & 1 & 0 & 0 & 0 & 0 \\
\hline WX & 22 & 66 & 82 & 42 & 94 & 30 & 37 & 41 & 40 & 44 & 1 \\
\hline WY & 13 & 37 & 45 & 12 & 46 & 5 & 17 & 10 & 25 & 41 & 43 \\
\hline Total & 469 & 1285 & 903 & 888 & 2192 & 453 & 604 & 484 & 916 & 591 & 854 \\
\hline$\%$ Protected & 73 & 77 & 76 & 78 & 75 & 77 & 84 & 88 & 82 & 84 & 80 \\
\hline Swales & & & & & & & & & & & \\
\hline UNPROTECTED SWALES & & & & & & & & & & \\
N of 4 & 65 & 30 & 36 & 62 & 74 & 11 & 8 & 3 & 16 & 10 & 13 \\
\hline E6-7 & & 6 & & 34 & 53 & 2 & 3 & & 4 & 3 & 2 \\
\hline E4-6 & 21 & 27 & 50 & 55 & 137 & 2 & 3 & 0 & 1 & 3 & 17 \\
\hline E1 & & 35 & 16 & & 104 & 6 & 28 & 5 & 61 & 5 & 60 \\
\hline G & 2 & 7 & 0 & 3 & 6 & 0 & 0 & 0 & 0 & 0 & \\
\hline LMN & 21 & 27 & 24 & 5 & 42 & 12 & 1 & 3 & 5 & 7 & 2 \\
\hline OP & 22 & 84 & 3 & 5 & 25 & 21 & 1 & 3 & 9 & 2 & 3 \\
\hline QR & 24 & 73 & 82 & 93 & 156 & 68 & 60 & 42 & 83 & 60 & 107 \\
\hline EQR & & 33 & 27 & 25 & 40 & 7 & & & & & \\
\hline DT & & & & & & & & & & & 3 \\
\hline ST & 10 & 28 & 34 & 9 & 40 & 0 & 3 & 0 & 1 & 0 & 2 \\
\hline U & 5 & 0 & 0 & 4 & 0 & 0 & 0 & 0 & 0 & 0 & 0 \\
\hline V & 2 & 0 & 0 & 0 & 5 & 1 & 0 & 0 & 3 & 2 & 2 \\
\hline X & 172 & 382 & 298 & 298 & 727 & 136 & 115 & 65 & 195 & 108 & 214 \\
\hline Y & 27 & 23 & 25 & 22 & 25 & 23 & 16 & 12 & 18 & 16 & 20 \\
\hline Total & & & & & & & & & & \\
\hline$\%$ Unprotected & & & 3 & 11 & 2 & 4 & 4 & 5 & 6 & 2 \\
\hline Swales & & & & & & & & & \\
\hline
\end{tabular}


The most important goal of our studies with the CPSD Tiger Beetle is to obtain information that will contribute to the protection and recovery of this rare species. Understanding what factors regulate population size and changing dynamics is probably the most critical aspect of this goal. As a result of our long term studies with this beetle and additional experience with tiger beetles, we have become convinced that rainfall is the primary factor controlling population size and the changing dynamics. Included in these recent studies at CPSD are field experiments that have documented how supplemental watering patches within interdunal swale areas attract adults with a coincidental increase in oviposition, larval recruitment, larval activity, survival and developmental rate. Evidence has also demonstrated how increased rainfall and soil moisture increase prey abundance which contributes to increased larval survival and adult fecundity. However, our attempts to directly correlate adult numbers with rainfall amounts over the past 15 years have not been as conclusive. A problem in developing this evidence is the two year life cycle of the tiger beetle and the varied direct and indirect effects of rainfall/soil moisture on many life history parameters. An additional problem may be our use of rainfall data from the Kanab station which may or may not be closely correlated with what is actually occurring at the dunes. Kanab is the closest station available for use and should provide us general useful trends in annual rainfall over the years.

There is some evidence that our rainfall data has supported the positive effects of rainfall on the adult population (Table 3, Fig. 4). For example, the high adult numbers in 1996 and 2002 followed several prior years of high rainfall. Also, we have noted reduced adult numbers in 2003-2005 following low rainfall amounts in 2001 to 2003. Another complication for developing correlations is that the actual pattern of rainfall within a year may be more important than the total rainfall. For example, the low adult numbers in 2004 and 2005 might be explained by the low rainfall in the April-June period in 2002 and 2003 compared to most previous years. There was significant rain in April 2003 but this was early in the month when it may have had little positive effect on adult oviposition. Also, total rainfall (April-October) for 2002 and 2003 were the lowest of any year since the early 1990 's and with low spring amounts probably combined to explain the low numbers in 2003 and 2004. In contrast, the May and total rainfall from 1997 to 2000 included four of the highest rainfall years in the past 12 years and may have explained the build-up of larvae in the northern dune area. Larvae there peaked to high numbers in 1998 and 1999.

The rainfall amounts, both total and April-June in 2006, 2007 and 2008 have been significantly lower than any other three-year period, and consequently project continuing low adult number for at least the next two years. The lowest ever total rainfall for Kanab was recorded in 2008 ( 2.82 inches) and perhaps more important is that the rainfall for April-June $(0.22)$ was also the lowest ever. This compares with the previous respective lows of 5.15 in 1993 and 0.41 in 2002 . Rainfall in May, probably the most important month for recruitment, was only .05 in $2006, .04$ in 2007 , and .13 in 2008, a much lower 
total for this month than any other three - year period. Our research plan for the next 4 or more years is to put in place a large number of soil moisture probes at several depths over much of the dune field to track weekly and seasonal variations in soil moisture.

Fig. 4. Rainfall totals for April through June and April through October at Kanab, UT, 1992 to 2008. April-June is the critical period for oviposition and larval recruitment.

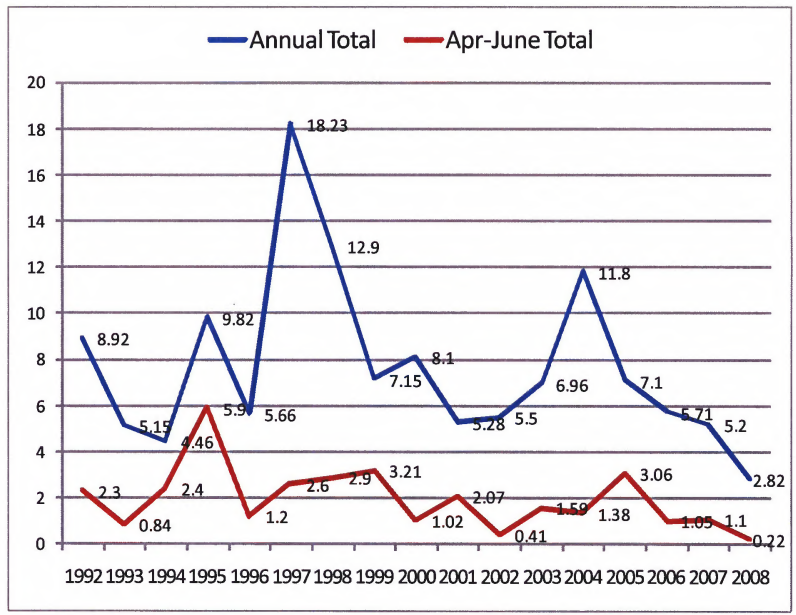


Table 3. Monthly rainfall at Kanab for periods when adults and larvae are active, April-June and April-October, 1992-2008.

\begin{tabular}{|c|c|c|c|c|c|c|c|c|c|c|c|c|c|c|c|c|c|}
\hline & 1992 & 1993 & 29 & 295 & 996 & 97 & 298 & 99 & 2000 & D01 & 2000 & 2000 & 20004 & 2000 & 200. & & \\
\hline$T$ & 0.17 & 0.06 & 2 & 2.2 & 0.43 & 0.89 & & 212 & Q.41 & 1.44 & 0.29 & 1 & 1.22 & 1.36 & 082 & 1.03 & 0.07 \\
\hline ay & 1.9 & 0.22 & 28 & 28 & 45 & 0.4 & & 0.5 & 08 & 51 & 12 & 59 & 0 & 49 & .05 & .04 & \\
\hline & 18 & 0.56 & Q11 & 0.9 & 03 & 1.34 & 0.45 & 0.84 & 253 & 0.12 & 0 & 0 & 0.16 & 21 & 18 & 2.08 & \\
\hline 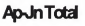 & 23 & 0.84 & 24 & 5.9 & 1.2 & 26 & 29 & 3.21 & 102 & 207 & .41 & 1.50 & 1.38 & 06 & .05 & 1.1 & \\
\hline & & .01 & 92 & 1.4 & 1.4 & 0.6 & 1. & 4.23 & 062 & 1.19 & 0.32 & 2.32 & 0.21 & 18 & 14 & 3 & 0.25 \\
\hline & 27 & 1.7 & 031 & 0.87 & 27 & 4.1 & 0. & 1.5 & 215 & 1.46 & 0.23 & 1.59 & 288 & 1.35 & 62 & 200 & \\
\hline & 94 & 0 & 054 & 1.7 & 0.91 & 9.1 & 5. & 1.4 & 027 & 0.64 & 256 & 0.7 & 1.67 & 0.01 & $a>8$ & 1.47 & \\
\hline & 21 & 26 & 1.2 & 0 & 1.9 & 1.8 & 2 & 0.02 & 404 & 0.27 & 1.98 & 0.76 & 564 & 25 & 212 & 0.01 & 65 \\
\hline & 67 & 4.31 & 207 & 97 & 4.48 & 56 & 10.1 & 7.15 & 7.08 & 3.56 & 5.09 & 5.37 & 10.4 & 4.04 & 466 & 41 & \\
\hline & 92 & 5.15 & 446 & 9.82 & 5.66 & 1823 & 129 & 7.15 & 81 & 5.28 & 55 & 6.96 & 11.8 & 7.1 & 571 & 2 & 82 \\
\hline tal & 227 & 131 & 113 & 249 & 144 & 463 & 328 & 182 & 206 & 132 & 75 & 177 & 299 & 180 & 145 & 132 & \\
\hline
\end{tabular}

\section{POPULATION VIABILITY ANALYSIS OF $C$. ALBISSIMA AND IMPLICATIONS FOR MANAGEMENT}

\section{Introduction}

Population viability analyses are an increasingly important tool for studies of the conservation of rare species. PVA models have been used to assist in identifying the threats faced by a species and evaluating the likelihood that the species will persist for a given time into the future. A PVA may be used to address three aspects of threatened species management: 1 . It may reveal that a population's long term viability is insensitive to certain population parameters and research may be directed at parameters that do have an impact on potential extinction; 2 . It may be used to assess the vulnerability of a species to extinction, and consequently, results of the analysis may be used to set policies and priorities by conservation groups; and 3. It may be used to predict potential effects of reintroduction, captive rearing, habitat manipulation, and other management strategies.

This part of the 2008 Annual Report presents a second population viability analysis (PVA) for the Coral Pink Sand Dunes Tiger Beetle. This updated version of the PVA includes the same basic model structure and the same parameters as the 1999 version, but is based on new parameter values which have been obtained by the population studies in the past 10 years, 1999-2008. The first PVA was based on data on the population from 1992 to 1998 . Since the first PVA, we have used population estimates that are more accurate and thus more reliable for determining inputs for this revised PVA. These recent studies have determined that the population estimates used in 
that previous PVA overestimated adult numbers in many of those years and were thus less reliable. Also, in part because of these new estimates and also because the true population size has been lower in these past 10 years than in the previous 7 years, a new model is very important for re-evaluation of extinction probabilities which will thus provide valuable input for the revision to the Conservation Agreement.

\section{METHODS}

\section{Background}

PVA's are usually developed from age- or stage-based population models requiring detailed and accurate input data, such as transition and survival probabilities between life stages and stage specific fecundity rates. Because much of this information is not available for the CPSD tiger beetle, an alternative method based on empirical estimates of adult population size obtained each year from 1999-2008. In many regards this method is quite appropriate to use and may provide a more accurate assessment than stage-based models because our population estimates were determined to be very accurate and the cumulative errors that may accrue from the multiple parameters in these other models are absent.

\section{Modeling Approach}

The CPSD tiger beetle population was assumed here to consist of one panmictic population, based on field observations over the years of study, 1992-2008. A small satellite population is found in the northern part of the dune field, but is considered to be a "sink" that contains excess individuals that have dispersed from the main southern population concentrated within Conservation Area B during periods of high density. Consequently, the model did not use a metapopulation approach which is common to many other tiger beetle species which have multiple populations.

In selecting a population model, we analyzed the observed population dynamics of the tiger beetle population in its primary habitat and calculated the key population parameters to be used in the model. As indicated above, unlike the previous PVA, we based our input parameters on annual population estimates from 1999 through 2008. These estimates were obtained using a removal method and program Capture which consistently provided low confidence intervals and thus accurate estimates of the populations each of these years. Mean population size was calculated to be 1227 individuals, with total population estimate being as low as 558 and as high as 2944 over the 10 years (Table 1). These values were analyzed to determine if the population exhibited density dependent fluctuations. Because the beetles exhibit a two-year life cycle, density dependence would occur on a two year interval. If density dependent effects influenced population dynamics, the slope of a regression of population abundance in year $t\left(N_{t} \log\right.$-transformed) on the rate of growth between year $t$ and year $\mathrm{t}$ $+2\left(\mathrm{~N}_{\mathrm{t}+2} / \mathrm{N}=\operatorname{lambda}, \log\right.$ transformed $)$ should be negative. The slope was not significantly different than zero, indicating a lack of density dependence. However, the statistical power of the test was low due to the limited total number of years in the 
analysis ( 8 cohorts), and it is possible that density dependent effects operate in this population. Morever, it is apparent from experience with this species that limitations related to the amount and quality of habitat (due to climatic induced variations in food availability, OHV disturbance and other factors) do exert an influence on the population. Because of this, a logistic growth model with a ceiling carrying capacity was selected for the modeling.

\section{Selected parameters}

The calculated parameters used as the basic input parameters for the model based on the 1999-2008 population data and additional parameters included in various model runs are given in Table 2. As indicated above, the mean population size for the 10 year period was 1227 , and thus rounded to 1200 for the starting population size for all runs. The calculated mean growth rate was 1.058 so this was used as the basic value. Although growth rates of less than 1.0 were recorded for 7 of the 10 years, indicating a decline from one year to two years later (two year life cycle), we used only positive values of growth rate in the model runs since negative values would result in relatively rapid extinction. This is unrealistic for the history of this population which has obviously existed for a very long period of time in these dunes with erratic population fluctuations, so the growth rate has had to be on average over 1.0. Variation in growth rates is characteristic of insect populations which exhibit explosive growth during periods of favorable conditions and dramatic declines when conditions are unfavorable. In the various model runs we used the maximum growth rate that we observed (2.3) and also an intermediate value of 1.35 which we believe to be a value that is realistic for this population.

Table 1. Population estimates and calculated cohort growth rates of the CPSD Tiger Beetle Population, 1999-2008.

\begin{tabular}{|r|r|r|}
\hline & Population & Growth \\
\hline Year & Estimate & \multicolumn{1}{c|}{ Rate } \\
\hline 1999 & 1804 & 0.81 \\
\hline 2000 & 1270 & 2.32 \\
\hline 2001 & 1459 & 0.41 \\
\hline 2002 & 2944 & 0.3 \\
\hline 2003 & 595 & 0.94 \\
\hline 2004 & 757 & 1.47 \\
\hline 2005 & 558 & 1.25 \\
\hline 2006 & 1112 & 0.96 \\
\hline 2007 & 700 & \\
\hline 2008 & 1072 & \\
\hline mean & 1227 & \\
\hline
\end{tabular}


One of the most important aspects in PVA is to account for the effects of stochastic events on population growth rate. This is especially true for invertebrate populations wherein temporal variation in abundance is probably due more to stochastic events in the environment than to density dependent effects. Environmental stochasticity was incorporated into the model by making cohort population growth rate a random variable. For each specified lambda (growth rate) the cohort growth rate for a particular time step in the model was selected from a normal distribution with mean growth rate and standard deviation equal to the value specified by the user. Larger specified values of the standard deviation in the model mean greater potential for any given year to be extremely poor or extremely good in terms of population growth rate. Appropriate values for the standard deviation of the growth rate were based on observed variation in cohort growth rates between 1999-2008. The standard deviation in cohort growth rate was calculated on an annual basis (i.e., as if odd and even year cohorts did not exist). This value, .646, was used as the base value in the model, but to model a broader range of potential stochasticity over time, we selected a lower value of .33 and a higher value of .95 as additional parameter values in various model runs.

The effects of carrying capacity $(\mathrm{K})$ on model predictions were examined by varying $\mathrm{K}$ in the model $(\mathrm{K}=800,1200,2000,3500,5000)$, while holding other parameters constant. The base value for carrying capacity in the model was set at 3500 , a value that was slightly higher than the maximum population size we have ever recorded, 2944. We increased this to 3500 to account for some additional adults that would likely be missed in any estimate, as we believe to be the case.

Table 2. Parameter values used in this PVA and those used for the previous PVA.

\begin{tabular}{|l|c|c|c|c|c||c|}
\hline & \multicolumn{3}{|l|}{$\begin{array}{l}\text { Base Values } \\
\text { from 1999-2008 Data }\end{array}$} & \multicolumn{4}{|l||}{ Altemative Values Used } & $\begin{array}{l}\text { Base Values } \\
\text { 1992-1998 Data }\end{array}$ \\
\hline Mean Growth Rate & $\mathbf{1 . 0 5 8}$ & 1.35 & 2.3 & & & $.86 / 1.5^{\star}$ \\
\hline Std Dev. & $\mathbf{0 . 6 4 6}$ & 0.33 & 0.95 & & & 0.95 \\
\hline Camying Capacity & $\mathbf{3 5 0 0}$ & 800 & 1200 & 2000 & 5000 & 3000 \\
\hline Starting Population & $\mathbf{1 2 0 0}$ & & & & & 2025 \\
\hline Ramas Models Used & Logistic with ceiling & Scramble & Contest & & & Logistic with ceiling \\
\hline
\end{tabular}

* 1.5 was selected as the most likely growth rate in the previous PVA. The calculated mean of 86 is a negative growth rate which would have resulted in rapid extinction.

Predicting Probability of Extinction Under Various Model Scenarios.

Program ECOLAB (Ramas Software, Inc.) was used to model population dynamics and extinction probability. The model ran for 50 time steps (equal to 50 cohorts and thus 100 years). At the beginning of each model run, the starting population size was specified as 1200 , the average population size for the past 10 years. Population abundance for the next time step was predicted based on the model's predicted lambda, 
generated from the normal distribution with specified standard deviation. This predicted population was then used in the next time step. The process was repeated for 50 time steps producing a time series of abundance for a simulated 100 year period. When a simulation was complete, original parameter values were reset to the specified initial conditions, and the model rerun. Predictions from each individual run, even if started from the same initial conditions, produced different abundance time series because of the environmental stochasticity incorporated into the model. In order to predict extinction probability under a specified set of initial conditions, 1000 replicate model runs were made. The percentage of runs that produced an extinction (population size reaching 0 at some point during the 100 year simulation) was the predicted probability of extinction for CPSD tiger beetles over the next 100 years. By varying initial conditions as indicated in Table 2, the effect of different model assumptions on predicted probability of extinction was evaluated.

It should also be pointed out that extinction of a population might also occur even before the population reached zero, as a result of Allee effects. Allee effects describe a situation where a population growth rate drops steeply, often to extinction, once a critically small population size is reached. For example, with too few individuals, finding mates may become difficult, leading to rapid decline in abundance. There is also evidence in many species that low population size of as little as 500 to 50 produces genetic decline due to loss of genetic diversity, inbreeding or related factors. Small populations are also more susceptible to extinction from catastrophic weather events or other natural or man-induced disturbances. Consequently, our results in all model runs show extinction risks to $0,50,100,200$ and 500 individuals.

\section{RESULTS AND DISCUSSION}

The results of the series of model runs using the range of parameters produced extinction rates from zero (no predicted chance of population extinction) to 1.0 (a $100 \%$ chance of extinction). Not surprising was that varying model parameters had dramatic effects on predicted extinction probability (see Table 3 ). This was determined by a sensitivity analysis in which one parameter was varied while holding the others constant. Several parameters, carrying capacity and starting population size had relatively little effect on extinction probability. For example, using the base values that we calculated for growth rate and standard deviation, probability of extinction was determined to be .32 $(=32 \%$ extinction probability) with a 3500 carrying capacity $(\mathrm{K}), .31$ with a $\mathrm{K}$ of 2000 , .35 if $\mathrm{K}$ was 1200 , and .37 if $\mathrm{K}$ was 800 (Table 3 ). Starting population size had even less affect although we do not present results here. Using two other models (logistic scramble and logistic contest) also did not produce appreciably different results (Table 3 ).

The two parameters which had very significant effects on extinction probabilities were growth rate and standard deviation (stochasticity) (Table 3, Fig. 1). For example, extinction probability is zero with growth rates of 1.35 and 2.3 (when stochasticity is .65 ) but .32 when growth rate is 1.06 . Extinction risk increases from zero to over .90 when stochasticity increases from .33 to .95 . The results of all combinations included in 
the PVA model runs are given in Table 3. In general these runs show that the CPSD tiger beetle population is at risk of extinction if growth rate remains at 1.058, the average for the past 8 cohorts), even when stochasticity is lower (.33) than we have recorded. By contrast, growth rates of 1.35 greatly reduce extinction risk, unless stochasticity is .95 . A growth rate of 2.3 , the highest recorded for any cohort, makes extinction unlikely, even when stochasticity is .95 (Table 3). The scenarios which we believe provides the "best guess" of a range of extinction risks are the one with the calculate parameters for 1999-2008 (1200 starting population, 3500 carrying capacity, .646 standard deviation or stochastacity measure, and 1.058 growth rate) and the other scenario with the same parameters except a growth rate of 1.35 . With a 1.058 growth rate, the probability of the population going to 0 was .32 , a high likelihood of extinction, but a 1.35 growth rate produced an extinction probability of only .001 . Additional output from the most likely parameter values in model runs that show population numbers over time and the full range of extinction probabilities are given in Fig. 2.

Fig. 1. Extinction risks resulting from different parameters. Probabilities that the population will reach 0, 50 or 100.

A. Extinction probabilities based on growth rate variations $(.65,1.3,2.3)$

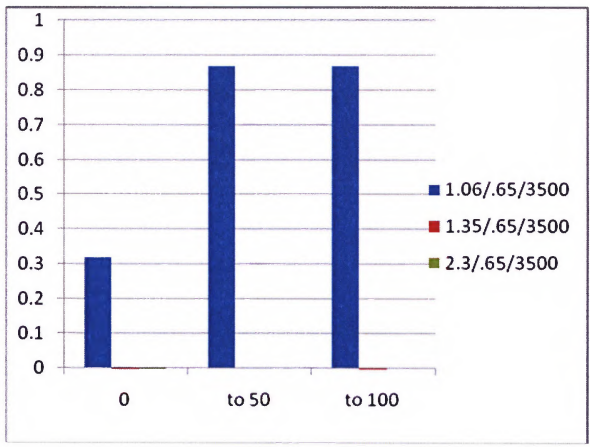




\section{B. Extinction probabilities based on stochasticity (standard deviation) variations.}

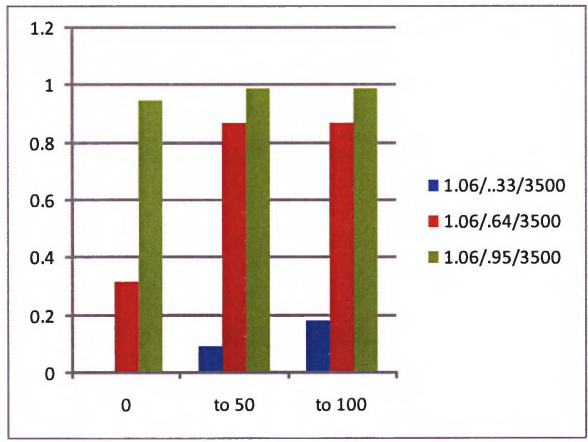

Table 3. Results of model runs using a range of parameter manipulations. Alb1, etc are designations for the respective parameters used. A starting population size of 1200 used in all runs. Note that Alb14 is comparable to the "best guess" input parameters for the previous PVA.

\begin{tabular}{|c|c|c|c|c|c|c|c|c|c|c|c|}
\hline & Ab1 & Alb2 & $A \mathrm{Ab3}$ & $\mathrm{Alb4}$ & Alb5 & $\overline{A b} 14$ & Alb6 & Alb7 & Alb8 & Alb17 & Ab18 \\
\hline Mean Growth Rate & 1.058 & 1.35 & 2.3 & 1.058 & 1.058 & 1.35 & 1.058 & 1.058 & 1.058 & 1.058 & 1.35 \\
\hline Std Dev. & 0.646 & 0.646 & 0.646 & 0.33 & 0.95 & 0.95 & 0.646 & 0.646 & 0.646 & 0.646 & 0.646 \\
\hline Carrying Capacity & 3500 & 3500 & 3500 & 3500 & 3500 & 3500 & 800 & 1200 & 2000 & 5000 & 5000 \\
\hline \multicolumn{12}{|l|}{ Prob. Popul will reach: } \\
\hline 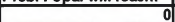 & 0.32 & 0.001 & 0.0001 & 0.002 & 0.95 & 0.007 & 0.37 & 0.35 & 0.31 & 0.27 & 0.001 \\
\hline 50 & 0.87 & 0.003 & 0 & 0.09 & 0.99 & 0.22 & 0.95 & 0.94 & 0.91 & 0.84 & 0.002 \\
\hline 100 & 0.87 & 0.002 & 0 & 0.18 & 0.99 & 0.36 & 0.95 & 0.94 & 0.91 & 0.91 & 0.009 \\
\hline 200 & 0.97 & 1,04 & 0 & 0.33 & 0.99 & 1 & 1 & 0.99 & 0.96 & 0.96 & 0.02 \\
\hline 500 & 0.99 & $\overline{0.23}$ & 0 & 0.63 & 1 & 1 & 1 & if & 1 & 0.99 & 0.16 \\
\hline
\end{tabular}

\begin{tabular}{|c|c|c|c|c|c|c|c|c|c|c|c|c|c|}
\hline & Alb11 & $\mathrm{Alb} 15$ & $\mathrm{Ab} 16$ & $\mathrm{Ab} 17$ & $\mathrm{Alb9}$ & Alb10 & $\mathrm{Alb12}$ & Ab13 & Alb1œr & $\sqrt[4]{162 c 0 n}$ & $\mathrm{Ab} 1 \approx \mathrm{C}$ & $\mathrm{Alb} 2 \mathrm{sc}$ & $\mathrm{Alb} 2 \mathrm{sc}$ \\
\hline Mean Growkh Rate & 1.35 & 23 & 23 & 1.058 & 1.35 & 1.35 & 2.3 & 1.058 & 1.058 & 1.35 & 1.058 & 1.35 & 1.35 \\
\hline Std Dev. & 0.33 & 0.95 & 0.33 & 0.646 & 0.646 & 0.33 & 0.646 & 0.95 & 0.646 & 0.646 & 0.646 & 0.646 & 0.646 \\
\hline Carrying Capacity & 3500 & 3500 & 3500 & 5000 & 1200 & 1200 & 1200 & 1200 & 3500 & 3500 & 3500 & 3500 & 35 \\
\hline \multicolumn{14}{|l|}{ Prob. Popu will reach: } \\
\hline 0 & 0 & 0 & 0 & 0.27 & 0.001 & 0 & 0 & 0.88 & 0.32 & 0.001 & 0.29 & 0.001 & 0.00 \\
\hline 50 & 0 & 0 & 0 & 0.84 & 0.012 & 0 & 0 & 0.99 & 0.9 & 0.03 & 99 & 0.05 & 0.05 \\
\hline 100 & 0 & 0 & 0 & 0.91 & 0.055 & 0 & 0 & 1 & 0.94 & 0.12 & 0.94 & 0.13 & \\
\hline 200 & 0 & 0 & 0 & 0.96 & 0.213 & 0 & 0 & 1 & 0.98 & 0.36 & 0.97 & 0.35 & \\
\hline 500 & 0.001 & 0.001 & 0 & 0.99 & 0.93 & 0.002 & 0.001 & 1 & 0.99 & 0.88 & 0.99 & 0.87 & \\
\hline
\end{tabular}


Fig. 2. Additional graphical representation of selected model runs.

A. Trajectory graph of population size over time for Alb 1(1.058, .646, 3500)

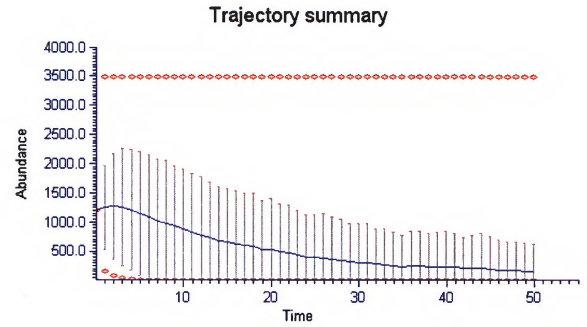

B. Probabilities population will reach a particular population size for Alb 1 (1.058, $.646,3500)$.

Extinction risk 0.319

Extinction/Decline

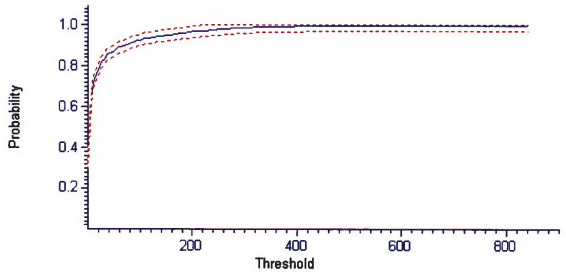


C. Trajectory graph of population size over time for Alb $2(1.35, .646,3500)$

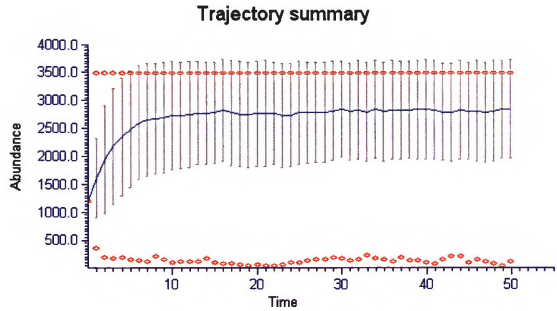

D. Probabilities population will reach a particular population size for Alb 2 (1.35, $.646,3500)$.

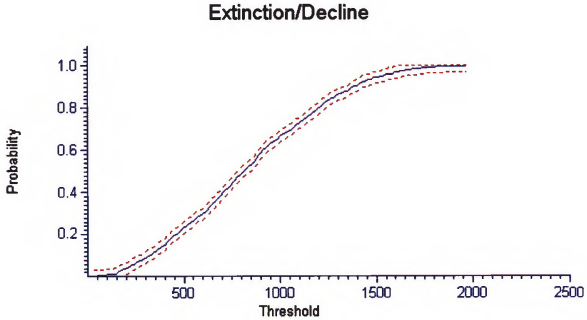


E. Trajectory graph of population size over time for Alb $14(1.35, .95,3500)$

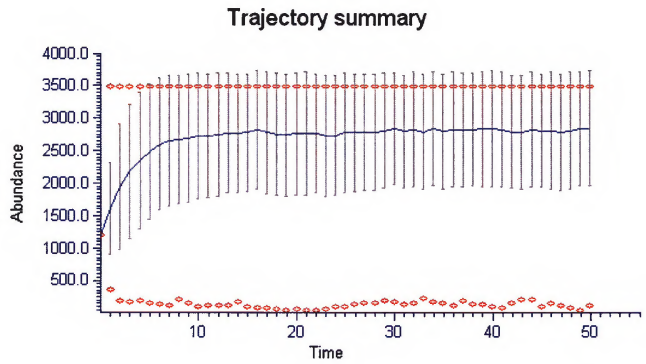

F. Probabilities population will reach a particular population size forh Alb 2 (1.35, $.646,3500)$.

Extinction risk $\sim 0.007$

Extinction/Decline

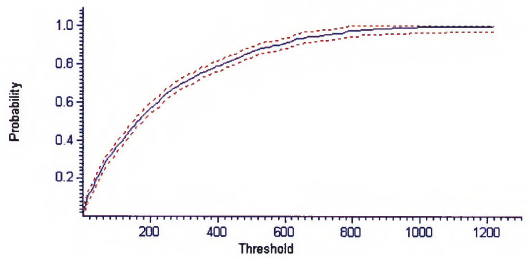




\section{SUMMARY AND M ANAGEMENT CONSIDERATIONS}

The results of this updated PVA indicates the CPSD tiger beetle is probably at even greater risk of extinction that projected in the 1999 PVA. The increased risk is essentially a result of the lower population sizes recorded in the past 10 years and the associated low annual growth rates during this period that drove the model results. The low growth rates resulting from low recruitment and survival during this 10 -year period are likely a result of low rainfall, especially in the past 5-6 years. Unfortunately, management options for increasing population size are limited because of the dominating effect of growth rates on population size. Since rainfall/soil moisture seems to be so important, supplementing moisture may offer an option for increasing ovipostion and recruitment, at least on a limited or localized basis. Our previous experiments demonstrated positive effects of moisture supplementation in localized areas. It is reasonable to conclude that larger scale moisture supplementation during the critical period of adult oviposition (May) in selected swales could have measurable effects, and will be carried out in 2009. A second management option that might have positive effects on growth rate by increasing oviposition and recruitment is to reduce vegetation cover in selected swales where it may have reduced oviposition sites in recent years. This option might also be implemented on a limited scale in 2009 by manual removal of vegetation (by clipping) is several swales. A third option that should be considered is an expansion of Conservation A to include several important swales that are believed to have suitable habitat, but are being impacted by heavy OHV use which prevents successful colonization and recruitment. One additional management option to increase growth rate and/or reduce extinction risk is to conduct captive rearing trials to produce first instar larvae for translocation into Conservation B in an attempt to establish an additional population there.

\section{Acknowledgments}

We greatly appreciate the assistance of Michael Franklin at Coral Pink Sand Dunes State Park who provided logistic support and provided accommodations during our field work at the sand dunes. The PVA work was done by Ryan Knisley and we greatly appreciate his assistance in this important part of the project. These studies we made possibly because of the interest and financial support through a Challenge Cost Share Agreement arranged by Mr. Ron Bolander of the Bureau of Land Management, Utah. 
